\title{
Methods for Diagnosing Some Malignant Neoplasms of the Canine Nasal Mucosa
}

\author{
Emilia BALINT $^{1 *}$, Florin DUMITRESCU ${ }^{1}$, Iuliana MIHAI ${ }^{2}$, Nicolae MANOLESCU ${ }^{1}$ \\ ${ }^{1}$ University of Agronomic Sciences and Veterinary Medicine of Bucharest, Faculty of Veterinary \\ Medicine -Splaiul Independentei no. 105 - sector 5 - postal code 050097, Bucharest - Romania \\ ${ }^{2}$ PhD Student of the Romanian Academy - Calea Victoriei 125, București 010071 \\ * corresponding author: emilia_balint@yahoo.com
}

Bulletin UASVM Veterinary Medicine 73(2)/2016

Print ISSN 1843-5270; Electronic ISSN 1843-5378

doi:10.15835/buasvmcn-vm:12229

\begin{abstract}
The aim of this study is to highlight the easiest method of investigation (nasal flush or rhinoscopy) depending on the animal condition. A total of 18 dogs were investigated, which were presented at the Faculty of Veterinary Medicine Bucharest, with respiratory distress due to obstruction of the nasal cavities. The sampling for cytological diagnosis has been made using the two techniques, the nasal flush and rhinoscopy. It is most useful to try the flush first, because it is less invasive, and only then, in case of poor cellularity after centrifugation, or in case of an inflammatory process with unfavourable post-treatment evolution, endoscopy will be used. Cytomorphological diagnosis, after rhinoscopy or nasal flush, has pinpointed the following forms of nasal cancer: 10 cases out of 18 were epithelial neoplasms of the olfactory mucosa; 6 cases out of 18 were nerve tumors (malignant melanoma, estesiocarcinoma); 2 cases out of 18 were mesenchymal tumors (osteosarcoma, fibrosarcoma). The nasal flush is a good sampling method but has the disadvantage of sometimes encountering an acute or chronic inflammatory process that can shield the tumor process.
\end{abstract}

Keywords: canine, cytomorphology, nasal neoplasm

\section{INTRODUCTION}

The literature debates the nasal cancer problems in dogs on many areas of the nasal mucosa histological structures. A sample of the mass via biopsy and histopathology (looking at the wedge of tissue under the microscope) or aspirate and cytology (looking at cells on a slide) is needed to determine what type of cancer is present (Masson, 1956). We have focused our attention on the cytomorphological aspects, which we have been correlated with the anatomo-clinical and imagistic ones, using two methods: nasal flush and rhinoscopy (Balint et al., 2015; AJCC, 2010).

Rhinoscopy involves insertion of a rigid or flexible fiberoptic viewing scope through the nostril into the nasal cavity. A flexible scope can also be inserted into the back of the mouth and retroflexed to examine the posterior part of the nasal cavity above the soft palate. The presence of fluid, masses, and any distortion of the tissues lining the nasal cavities are documented. If any abnormality is located, a biopsy of the tissue can be taken with special biopsy forceps. Most of the nasal cavity in medium to large dogs can be visualized. Some limitations exist in cats and smaller dogs because their nasal cavities are so small. On occasion, the frontal sinuses can be accessed and evaluated; however, this is not routinely possible. Use of a flushing solution to enhance visualization and a rigid endoscope instead of a flexible one may increase the chances of achieving adequate examination of the front part of the nasal cavities, but a flexible scope is better at examining the posterior nasal cavity (12). 
The purpose of the study is to analyze the possibility to choose the most useful method of cytological diagnosis between the two of them (nasal flush and rhinoscopy), based on the clinical aspects of the investigated animal regarding the advantages and disadvantages of each technique (Cummings, 2010; Lo et al., 2010; Lo et al., 2012; Lo et al., 2013; Lozano, 2012; Manolescu and Balint, 2010).

\section{MATERIALS AND METHOD}

There were 18 dogs investigated, whom were presented with their owners at the Veterinary Clinic for the Faculty of Veterinary Medicine in Bucharest, with respiratory problems due to obstructions of the nasal cavity.

Clinical, they presented facial deformations in the nasal or sinusal area and/or bloody fluid loss with different grades of respiratory difficulty.

The cases that involved rhinal loss of bloody fluids, the nasal cavity was flushed with saline solution. The material resulted following centrifugation (1500 RPM/10 min) was applied on slides, using panoptic staining (May-Grunwal Giemsa). The cytomorphologic diagnose was performed using Olympus BX 51 optical microscope.

The rest of the cases that did not allow the nasal flush, were approached with an endoscopy exam by a specialist veterinary physician; if the animal presented facial bone deformations a radiological exam was performed.

The rhinoscopy is a high value component in the nasal cavity examination and completes itself with other specialty exams.

\section{RESULTS AND DISCUSSIONS}

The cytomorphologic diagnosis was obtained through two techniques, nasal flush and rhinoscopy. The most useful method is the first one, being easier to accomplish, less invasive and with a $75 \%$ reliability. In case of an inflammatory process within the cytomorphological exam we can tell whether it evolves acute or chronic in order to establish the therapy. Just in case of lack of cellularity after the centrifugation or by diagnosing an inflammatory process with adverse post-therapy evolution, endoscopy will be used. In this case, the inflammatory process can go with a neoplastic process.

Endoscopy is the most secure method for establishing the nasal mucosa pathology diagnosis. However, it is a laborious technique, presenting risks arising from general anesthesia, and the occurrence of postoperative bleeding. Therefore, it is recommended to first try getting the diagnostic using the flush technique (Tams and Rawlings, 2010).

\section{The distribution of the cytomorphological forms of nasal and sinusal neoplasms}

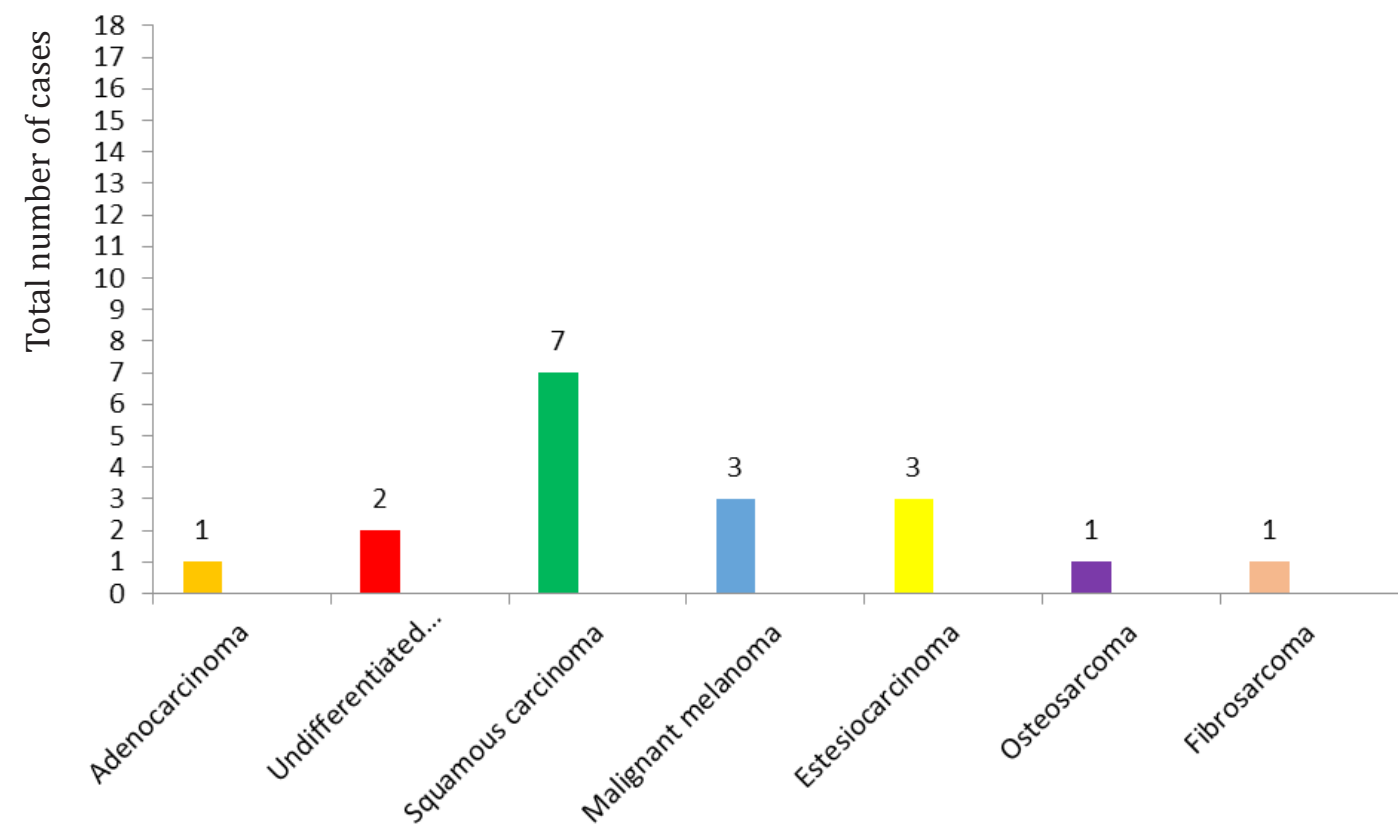


The morphocytology diagnosis revealed the following types of nasal cancer:

- 10 of the 18 cases turned out to be epithelial neoplasms of the olfactory mucosa:

- 1 of the 10 cases revealed the malignization of the glandular cells responsible for mucus production within the mucus glands (adenocarcinoma);

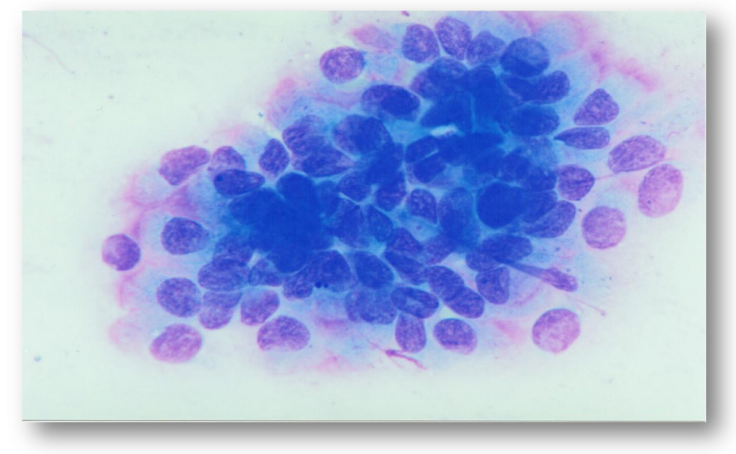

Fig. 1. Undifferentiated vegetant carcinomaMGG $\mathbf{x}$ 1000 - classic "finger glove" aspect

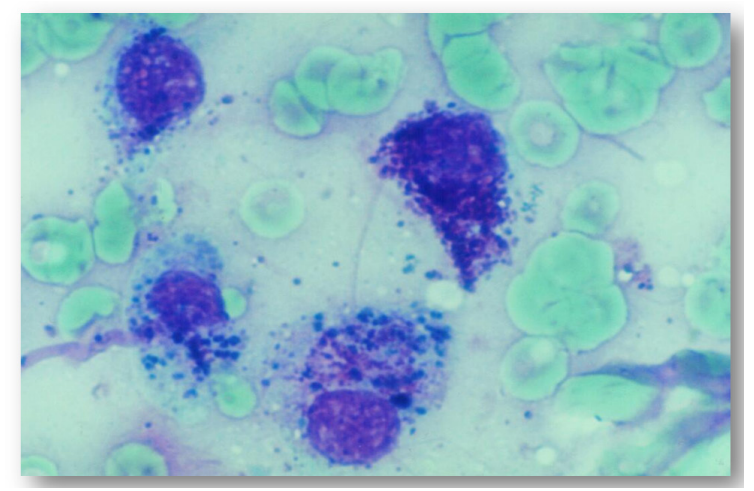

Fig. 3. Malignant melanoma of the nasal mucosa

MGG x 1000. Exclusively proliferation of tumor cells loaded with melanin pigment (melanocytes).

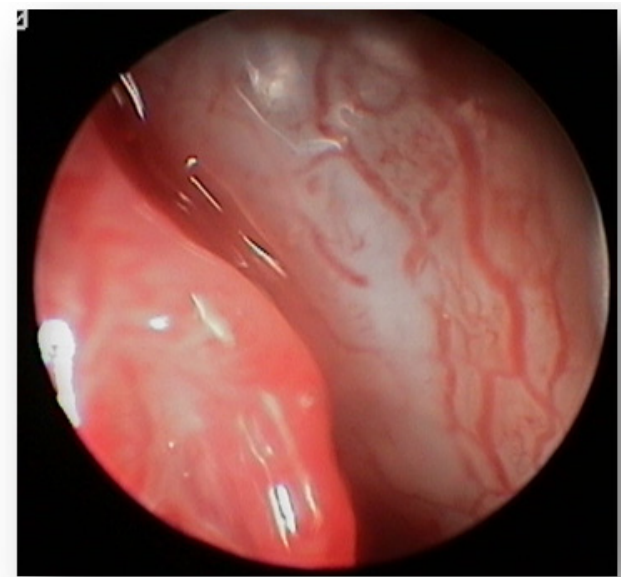

- 9 cases were undifferentiated vegetant or squamous carcinomas, resulted from the malignization of the respiratory epithelium; - 6 out of the 18 cases turned out to be nervous system tumors, either by the malignization of the melanic cells present in the submucosa, generating a typical malignant melanoma, or by the malignization of the sensorial nervous cells from the nasal respiratory mucosa, generating the aspect of estesiocarcinoma.

- 2 out of 18 cases turned out to be mesenchymal tumors: 1 osteosarcoma and 1 fibrosarcoma.

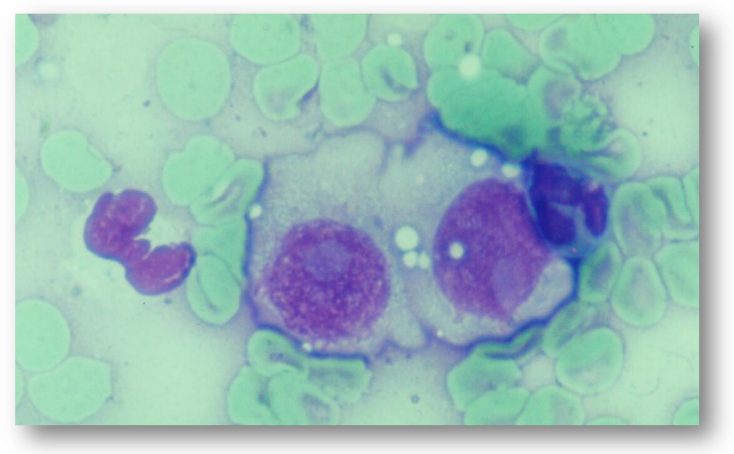

Fig. 2. Invasive adenocarcinoma of high malignancy - MGG x 2000. It identifies in every cell of he corionic glands a high atipism, with monstrosities, cytoplasmic basophilia and the presence of giant nucleolus.

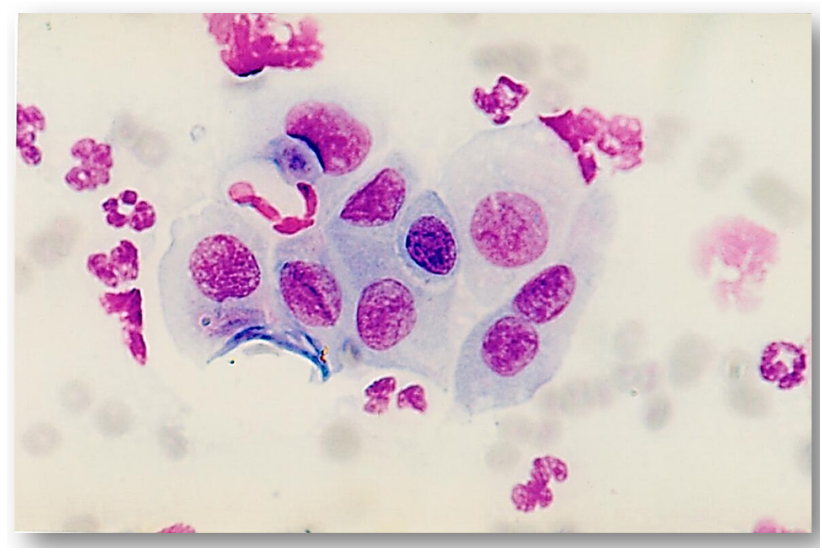

Fig. 4. Esteziocarcinoma of the nasal mucosa MGG x 1000. It identifies sensory epithelial cells with malignant cell aspect.

Fig. 5. Left nostril (rhinoscopy) - Notice a swollen, discolored area in the nose cone. Cytomorphological diagnosis: esteziocarcinoma. 


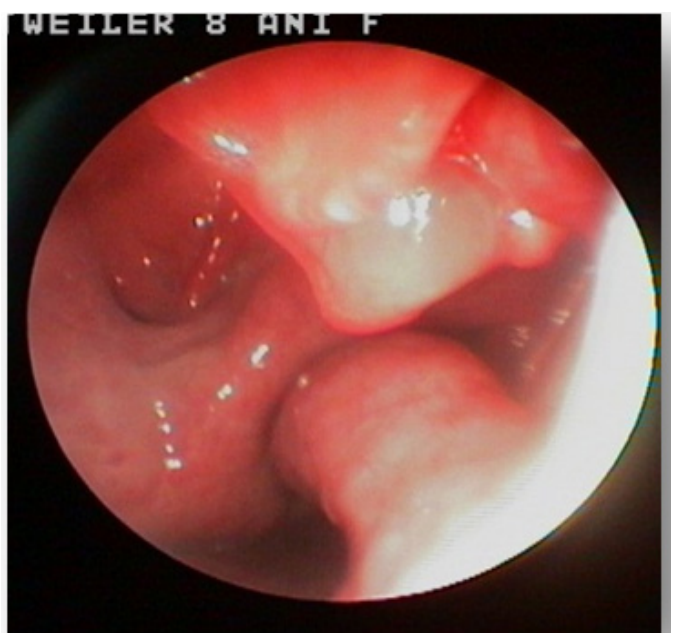

Fig. 6. Right nostril (rhinoscopy) - The lumen is seen almost nonexistent and a portion of the cone hypertrophied, with a white-hyperemic aspect, different from the rest of the mucosa. Cytomorphological diagnosis: high malignant adenocarcinoma

\section{CONCLUSIONS}

The clinical symptoms of nasal neoplasm have an early onset and are extremely specific;

The intra-nasal imaging exam (rhinoscopy), followed by the biopsy exam, is an excellent means of diagnosis of the nasal neoplasms.

The nasal lavage, in the case of bloody leaks, is also a good means of diagnosis but has the disadvantage of sometimes presenting, at the morphocytology exam, an acute or chronic inflammatory process that can shield the tumor process.

The morphocytology exam performed through rhinoscopy precisely establishes the differential diagnostic between an acute rhinitis and a neoplasm.

\section{REFERENCES}

1. American Joint Committee on Cancer (AJCC) (2010) Cancer Staging Manual. 7th ed. New York, NY: Springer, pp 41-56.

2. Balint E, Manolescu N, Lastofka D (2015). Cytomorphological aspects of some otho-rhino-laringology cancers in the canine and feline. Procedings of the Romanian Academy, vol. 6.

3. Cummings (2010). Otolaryngology. 5th ed, Chapter 99. pg 1344.

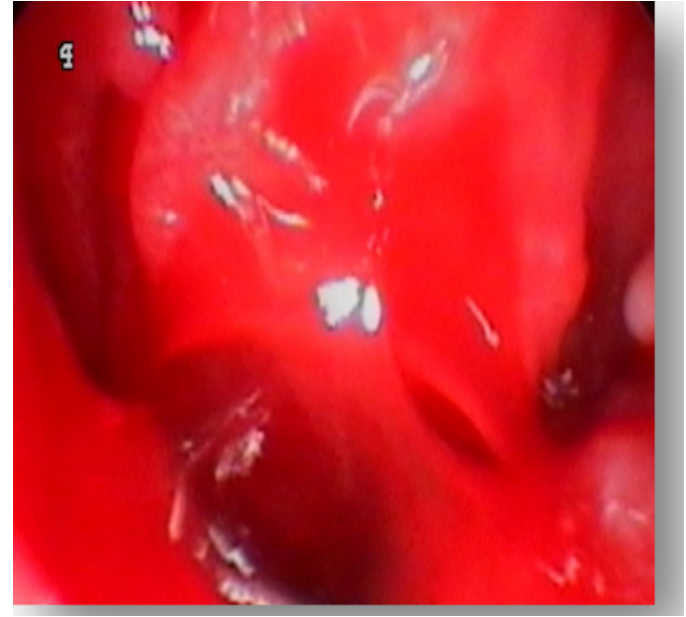

Fig. 7. Left nostril (rhinoscopy) - Particularly pronounced hypertrophy. Notice a portion of the nose cone hypertrophied, that almost completely obliterates the lower nasal meatus. Cytomorphological diagnosis: nasal carcinoma

4. Lo AK, Lo KW, Ko CW et al. (2013). Inhibition of the LKB1AMPK Pathway by the Epstein-Barr Virus-encoded LMP1 Promotes Proliferation and Transformation of Human Nasopharyngeal Epithelial Cells. J Pathol.

5. Lo EJ, Bell D, Woo JS, et al. (2010). Human papillomavirus and WHO type I nasopharyngeal carcinoma. Laryngoscope. 120(10):1990-7.

6. Lo KW, Chung GT, To KF. (2012). Deciphering the molecular genetic basis of NPC through molecular, cytogenetic, and epigenetic approaches. Semin Cancer Biol. 22(2):79-86.

7. Lozano R (2012). Global and regional mortality from 235 causes of death for 20 age groups in 1990 and 2010: a systematic analysis for the Global Burden of Disease Study 2010. Lancet 380 (9859): 2095-128.

8. Manolescu N, Balint E (2010). Atlas of Canine and Feline Oncocytomorphology, Curtea Veche, Publishing, Bucharest.

9. Tams TR, Rawlings CA (2010). Small animal endoscopy. 3rd edition, 2010, Elsevier Health Sciences.

10. Pierre Masson (1956). Human Tumors: Histology, Diagnosis, and Techniques, second edition, Books on Demand

Web sources:

11. http://wiredpen.com/wp-content/uploads/2016/06/ vet-rochester-nasal-disease-canine.pdf

12. http://www.saintfrancis.org/wp-content/uploads/ Rhinoscopy.pdf 